\title{
Effect of sodium-glucose cotransporter type 2 inhibitors on liver fat in patients with type 2 diabetes: hepatic beyond cardiovascular and renal protection?
}

\author{
André J. Scheen ${ }^{1,2}$ \\ ${ }^{1}$ Division of Clinical Pharmacology, Centre for Interdisciplinary Research on Medicines (CIRM), University of Liège, Liège, Belgium; ${ }^{2}$ Division of \\ Diabetes, Nutrition and Metabolic Disorders, Department of Medicine, CHU Liège, Liège, Belgium \\ Correspondence to: Professor André J. Scheen. Department of Medicine, CHU Sart Tilman (B35), B-4000 LIEGE 1, Belgium. \\ Email: andre.scheen@chuliege.be. \\ Provenance: This is an invited Editorial commissioned by the Section Editor Jianqing Tian (Department of Endocrinology, Xiamen Humanity \\ Hospital, Xiamen, China). \\ Comment on: Kuchay MS, Krishan S, Mishra SK, et al. Effect of Empagliflozin on Liver Fat in Patients With Type 2 Diabetes and Nonalcoholic Fatty \\ Liver Disease: A Randomized Controlled Trial (E-LIFT Trial). Diabetes Care 2018;41:1801-8.
}

Submitted Oct 08, 2018. Accepted for publication Oct 12, 2018.

doi: 10.21037/atm.2018.10.39

View this article at: http://dx.doi.org/10.21037/atm.2018.10.39

Non-alcoholic fatty liver disease (NAFLD) is a common finding in obese people, especially patients with type 2 diabetes (T2DM). It may progress to non-alcoholic steatohepatitis (NASH) and ultimately fibrosis and cirrhosis (1-3). Because of the epidemics of both obesity and T2DM, the prevalence of NAFLD will increase, potentially causing a tremendous clinical and economic burden (4). Recent data showed that NAFLD is not only confined to liverrelated morbidity and mortality, but rather should be considered as a multisystem disease, as it increases the risks of cardiovascular disease and chronic kidney disease (5). The role of low-grade inflammation appears crucial in all these comorbidities, a finding that may be targeted by some glucose-lowering agents (6), including sodium-glucose cotransporter type 2 (SGLT2) inhibitors (7).

There is no approved pharmacotherapy for patients with NAFLD and NASH (2), yet numerous pharmacological strategies have been evaluated in clinical studies or are still in current development $(3,8)$. Among antidiabetes medications, besides pioglitazone (a thiazolidinedione acting as insulin-sensitizer) and liraglutide [a glucagon-like peptide-1 (GLP-1) receptor agonist], SGLT2 inhibitors have shown some efficacy in early preliminary experimental and clinical studies devoted to NAFLD $(8,9)$. Considering their positive impact on cardiovascular events (10) and renal outcomes (11), SGLT2 inhibitors occupy an increasing role in the management of T2DM (12). In this context, their effects on NAFLD raise increasing interest and certainly deserve further investigation.

The E-LIFT ("Effect of Empagliflozin on Liver Fat Content in Patients with Type 2 Diabetes") study was an investigator-initiated prospective, open-label randomized clinical trial (RCT) to study the effect of the SGLT2 inhibitor empagliflozin in T2DM patients with NAFLD (13). Fifty patients were randomly assigned to either the empagliflozin group (standard treatment for T2DM plus empagliflozin $10 \mathrm{mg}$ daily) or the control group (standard treatment without empagliflozin) for 20 weeks. Change in liver fat was measured by using MRIderived proton density fat fraction (MRI-PDFF), a robust and quantitative biomarker of hepatic steatosis (intracellular fat accumulation in hepatocytes). Secondary outcome measures were changes in serum liver enzymes, i.e., alanine transaminase (ALT), aspartate transaminase (AST), and gamma-glutamyl transferase (GGT) levels. When added to the standard treatment for T2DM, empagliflozin was significantly better at reducing liver fat (mean MRI-PDFF difference between the empagliflozin and control groups at 20 weeks: $-4.0 \% ; \mathrm{P}<0.0001)$. Compared to baseline, significant reduction was found in the end-of-treatment liver fat content (MRI-PDFF) for the empagliflozin group while a non-significant change was found in the 
Table 1 Comparison of the effects of SGLT2 inhibitors versus placebo on liver enzymes and measurements of liver fat content in patients with T2DM included in randomized controlled trials

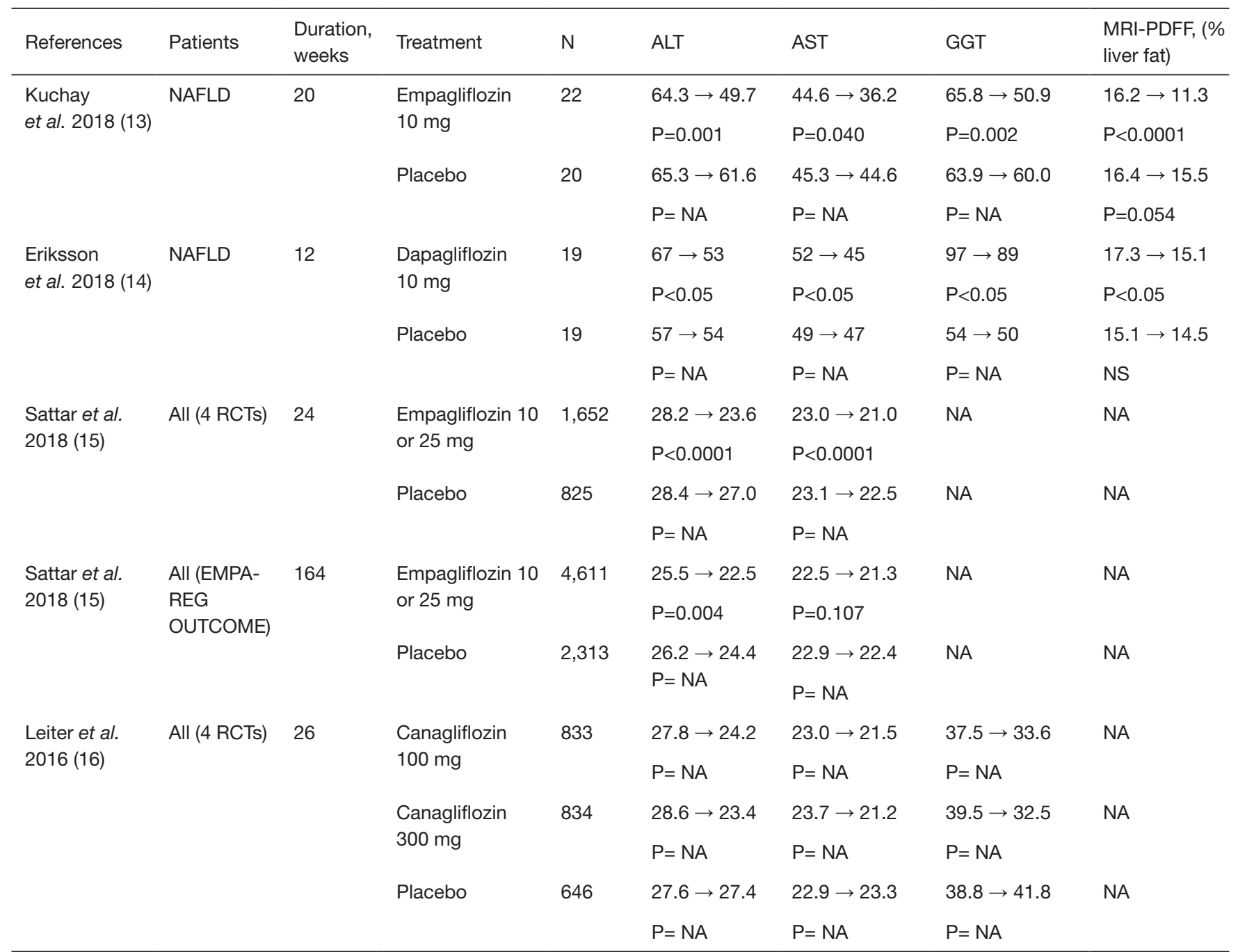

Results are expressed as changes before $\rightarrow$ after treatment. Open non-controlled studies are not considered in this table. MRI-PDFF, magnetic resonance imaging-proton density fat fraction; NA, not available; NS, not significant; NAFLD, non-alcoholic fatty liver disease; RCTs, randomized controlled trials; ALT, alanine transaminase; AST, aspartate transaminase; GGT, gamma-glutamyl transferase.

control group (Table 1). The empagliflozin group showed a significant reduction in serum ALT level and a numerical reduction in AST and GGT levels. The conclusion was that empagliflozin, added to standard treatment, reduces liver fat and improves ALT levels in patients with T2DM and NAFLD (13).

Interestingly, similar results were reported with dapagliflozin in T2DM patients with NAFLD (14). In a 12 -week RCT, the SGLT2 inhibitor reduced the liver fat content, again assessed by MRI-PDFF and used as primary endpoint, by $13 \%$ compared to placebo
(Table 1). Dapagliflozin monotherapy also reduced all measured hepatocyte injury biomarkers, including ALT, AST and GGT, and fibroblast growth factor 21 (FGF21), suggesting reduced cell damage and improved mitochondrial function or reduced endoplasmic reticulum stress associated with NAFLD. Furthermore, it showed that combined treatment of dapagliflozin with omega-3 (n-3) carboxylic acids further reduced liver fat content $(-21 \%$ with combined therapy versus $-13 \%$ with dapagliflozin monotherapy) (14).

Several other recent studies compared the effects of 
SGLT2 inhibitors on liver biomarkers either with placebo (Table 1) or with other glucose-lowering agents (Table 2). Two types of population were analysed, patients with not well controlled T2DM (but not screened for NAFLD) having participated to large RCTs and patients with T2DM specifically selected upon the presence of NAFLD at baseline in RCTs of smaller size. These studies used different complementary approaches to assess the presence and the severity of NAFLD $(2,22)$. All used clinical chemistry, i.e., serum liver enzymes as indirect markers of steatosis, and some of them also added imaging techniques in order to assess hepatic fat content. Instead of using MRI data, as in the two above-described studies $(13,14)$, CT scans were performed to assess the liver/spleen attenuation ratio as an indirect marker of NAFLD (the lower the ratio, the higher the degree of NAFLD). Some studies also used transient elastography (FibroScan ${ }^{\circledR}$ ) to assess the degree of fibrosis in complement to the FIB4 fibrotic index derived from biological and clinical measurements (18). Yet only one pilot study used liver biopsy (23), which is considered as the gold standard method providing the most detailed pictures of NAFLD, NASH and fibrosis $(2,22)$. This unique prospective open-label study based on serial liver biopsies demonstrated that canagliflozin improves the rates of hepatocyte steatosis and NAFLD activity score at 24 weeks in all five patients together with improvement in histopathologic findings (23).

Compared to placebo, positive results were reported with empagliflozin $(13,15)$, dapagliflozin (14) and canagliflozin (16) (Table 1). In post-hoc analyses, some significant, although small, reductions in serum liver enzymes were already observed with SGLT2 inhibitors in non-selected patients with T2DM insufficiently controlled on baseline therapies having participated to large phase III RCTs $(15,16)$. Of note, reductions were more marked in T2DM patients specifically selected for having NAFLD randomized to RCTs of smaller size $(13,14)$. No imaging assessment of liver fat content was performed in these placebo-controlled studies, except in the two studies using MRI-PDFF measurement $(13,14)$.

As compared with metformin $(21)$, glimepiride $(15,19)$ and dipeptidyl peptide-4 (DPP-4) inhibitors (16), SGLT2 inhibitors (luseogliflozin, canagliflozin, empagliflozin) were associated with significant reductions in serum liver enzyme levels (mainly ALT), despite similar glucose control (Table 2). These results were confirmed in two studies comparing dapagliflozin with other glucose-lowering medications $(17,18)$ and in a meta-analysis of RCTs with canagliflozin (24). When comparing the effects of canagliflozin versus sitagliptin or versus placebo, the overall differences in ALT and AST were almost similar in favour of the SGLT2 inhibitor (16). A similar conclusion may be drawn when comparing the effects of empagliflozin on ALT and AST changes in studies where glimepiride or placebo were used as controls (15) (Tables 1,2). In two studies, a reduction in liver fat content as assessed by a significant increase in liver/spleen attenuation ratio was observed with SGLT2 inhibitors compared with other glucose-lowering agents $(17,21)$ (Table 2). In all studies, some weight loss and, when body composition was assessed, a reduction in fat mass and visceral adipose tissue were noticed in patients treated with SGLT2 inhibitors compared with those receiving other antidiabetic agents $(14,17,18,20,21)$. Compared with pioglitazone, a compound that has proven its efficacy in reducing fat liver content in patients with T2DM and NAFLD $(2,22)$, ipragliflozin exerted equally beneficial effects on NAFLD markers and glycemic control in patients with T2DM complicated by NAFLD, but significantly reduced body weight and abdominal fat area (25). To our knowledge, no RCT compared the effects of a SGLT2 inhibitor with those of the GLP-1 receptor agonist liraglutide, another compound that has proven its efficacy in reducing liver fat content in T2DM patients with NAFLD $(2,15)$. In a large observational study using the database from a Canadian diabetes register, changes in serum levels of ALT, the most specific liver enzyme for NAFLD, were measured after a mean follow-up of 4.8 months in a total of 3,667 patients with T2DM who had canagliflozin, dapagliflozin, liraglutide or sitagliptin added to their diabetes treatments (25). ALT levels were lower after treatment with SGLT2 inhibitors, canagliflozin $(-4.3 \mathrm{U} / \mathrm{L})$ and dapagliflozin $(-3.5 \mathrm{U} / \mathrm{L})$, compared to incretin agents, liraglutide $(-2.1 \mathrm{U} / \mathrm{L})$ and sitagliptin $(-1.8 \mathrm{U} / \mathrm{L})$, each greater than the control group $(\mathrm{P}<0.01$ versus no added treatment). Of note, only the SGLT2 inhibitor treatment groups maintained a significant ALT reduction versus control following multivariable adjustment and propensity score weighting. SGLT2 inhibitors canagliflozin and dapagliflozin resulted in a weight and HbA1c-independent reduction of ALT levels compared to incretin agents, with a dose-response observed at higher baseline ALT levels (25).

The reduction in serum liver enzyme levels may appear rather small, yet statistically significant, when considering the all T2M population. However, numerous T2DM patients included in these studies not specifically dedicated 


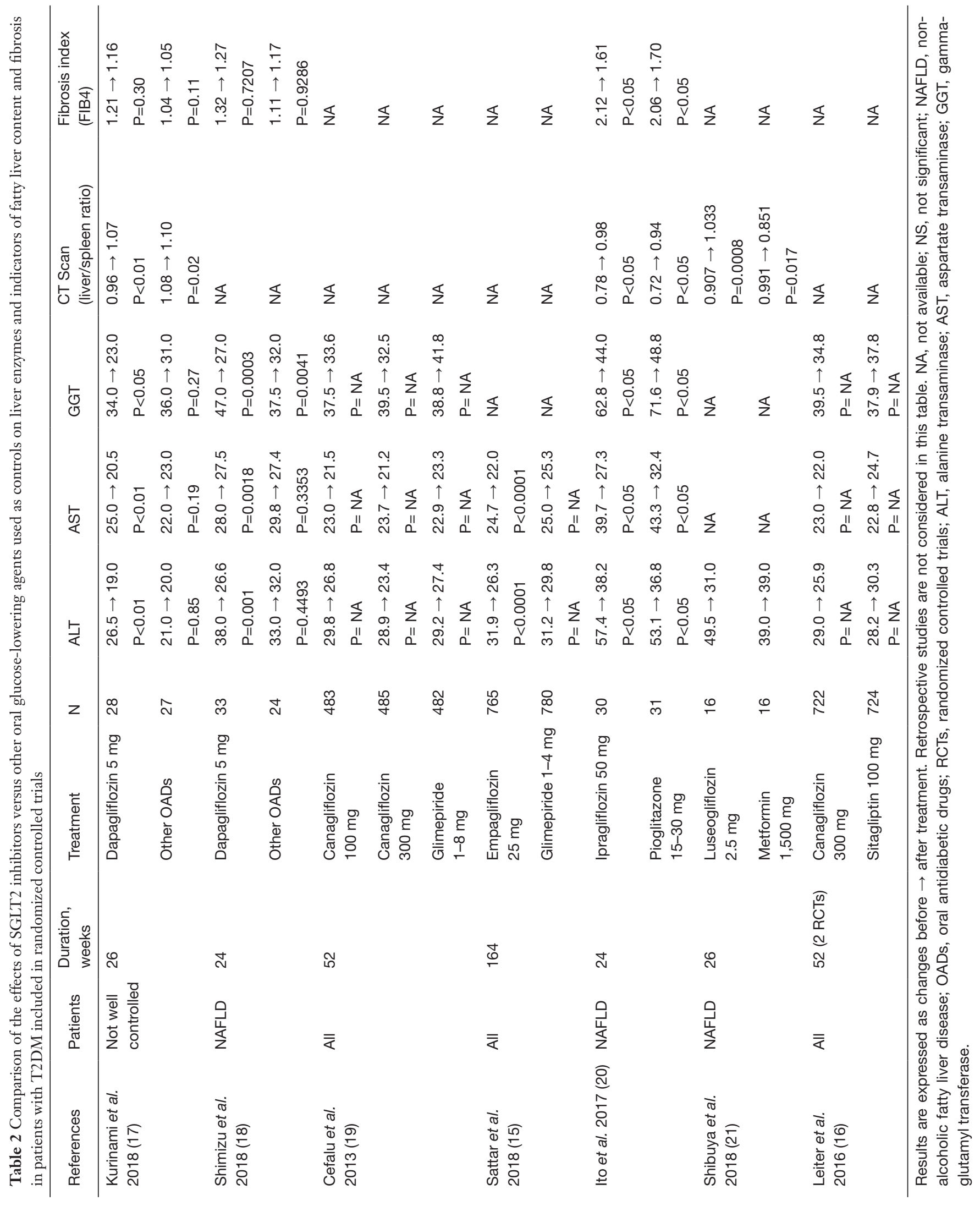


to NAFLD had normal levels at baseline. When the population was divided into tertiles according to baseline levels of ALT or AST, the reductions in serum liver enzymes observed with empagliflozin were consistently greater in the upper tertile subgroup (18).

In addition to analyse biomarkers of NAFLD, several of these studies investigated the effects SGLT2 inhibitors on fibrosis. Using the FIB4 index, they did not show any significant effect compared to baseline or compared with other glucose-lowering agents $(17,18)$. This apparent absence of effect on fibrotic process might be explained by a too short duration of these studies lasting 24-26 weeks only. Nevertheless, one study showed a significant reduction in FIB4 index with ipragliflozin at 24 weeks, similar to that observed with pioglitazone (20).

The underlying mechanisms responsible for improvement of NAFLD with SGLT2 inhibitors remain largely unknown and presently only speculations are possible. In one study, canagliflozin provided improvements in liver function tests versus either placebo or sitagliptin treatments that were fully explained by the combined effects of glycated hemoglobin (HbA1c) and body weight reductions with the SGLT2 inhibitor (16). However, in EMPA-REG OUTCOME RCT, the proportion of ALT-lowering effect of empagliflozin versus placebo that was considered independent of concomitant changes from baseline in HbA1c and body weight averaged $76.0 \%$ both after 24 and 164 weeks. In another study, improvement of liver dysfunction with ipragliflozin in patients with T2DM occurred irrespective of body weight reduction (9). Furthermore, in a study with MRI assessment of liver fat content, there was no significant correlations between liver fat reduction and improvement in HbA1c or body weight reduction (13). These observations may suggest the intervention of other subtle mechanisms. In this respect, the potential beneficial effects of SGLT2 inhibitors on low-grade inflammation and oxidative stress certainly deserve further investigations as recently discussed (7).

In conclusion, besides pioglitazone and liraglutide, SGLT2 inhibitors have shown emerging positive effects on NAFLD in patients with T2DM. These effects occur largely beyond the glucose-lowering activity as they are more marked than those observed with sulfonylureas, DPP4 inhibitors or even metformin, in patients reaching almost similar glucose control. The underlying mechanisms remain to be investigated beyond the effects on glycemia and body weight. Anti-inflammatory effects and reduction of oxidative stress may play a role. If thiazolidinediones, GLP-
1 receptor agonists and SGLT2 inhibitors all can reduce liver fat content in patients with T2DM and NAFLD and if these effects occur by different and potentially complementary mechanisms, one may speculate that combined therapy may be even more effective. This remains to be demonstrated in dedicated clinical RCTs that should selectively recruit T2DM patients with severe NAFLD. Additionally, it remains to be proven that the improvement of NAFLD with these compounds will be able to avoid the progression to NASH and ultimately fibrosis and cirrhosis. Finally, it is important to note that SGLT2 inhibitors as any other glucose-lowering agent have not the indication of improving NAFLD yet and further research is mandatory to progress in this evolving field.

\section{Acknowledgements}

None.

\section{Footnote}

Conflicts of Interest: The author has no conflicts of interest to declare.

\section{References}

1. Scheen AJ, Luyckx FH. Obesity and liver disease. Best Pract Res Clin Endocrinol Metab 2002;16:703-16.

2. European Association for the Study of the Liver (EASL), European Association for the Study of Diabetes (EASD), European Association for the Study of Obesity (EASO). EASL-EASD-EASO Clinical Practice Guidelines for the management of non-alcoholic fatty liver disease. J Hepatol 2016;64:1388-402.

3. Stefan N, Häring HU, Cusi K. Non-alcoholic fatty liver disease: causes, diagnosis, cardiometabolic consequences, and treatment strategies. Lancet Diabetes Endocrinol 2018. [Epub ahead of print].

4. Younossi Z, Tacke F, Arrese M, et al. Global perspectives on non-alcoholic fatty liver disease and non-alcoholic steatohepatitis. Hepatology 2018. [Epub ahead of print].

5. Byrne CD, Targher G. NAFLD: a multisystem disease. J Hepatol 2015;62:S47-64.

6. Scheen AJ, Esser N, Paquot N. Antidiabetic agents: Potential anti-inflammatory activity beyond glucose control. Diabetes Metab 2015;41:183-94.

7. Bonnet F, Scheen AJ. Effects of SGLT2 inhibitors on systemic and tissue low-grade inflammation: The potential 
contribution to diabetes complications and cardiovascular disease. Diabetes Metab 2018. [Epub ahead of print].

8. Sumida Y, Yoneda M. Current and future pharmacological therapies for NAFLD/NASH. J Gastroenterol 2018;53:362-76.

9. Komiya C, Tsuchiya K, Shiba K, et al. Ipragliflozin improves hepatic steatosis in obese mice and liver dysfunction in type 2 diabetic patients irrespective of body weight reduction. PLoS One 2016;11:e0151511.

10. Scheen AJ. Cardiovascular effects of new oral glucoselowering agents: DPP-4 and SGLT-2 inhibitors. Circ Res 2018;122:1439-59.

11. Scheen AJ. Effects of glucose-lowering agents on renal surrogate endpoints and hard clinical outcomes in patients with type 2 diabetes. Diabetes Metab 2018. [Epub ahead of print].

12. Davies MJ, D'Alessio DA, Fradkin J, et al. Management of Hyperglycemia in Type 2 Diabetes, 2018. A Consensus Report by the American Diabetes Association (ADA) and the European Association for the Study of Diabetes (EASD). Diabetes Care 2018. [Epub ahead of print].

13. Kuchay MS, Krishan S, Mishra SK, et al. Effect of empagliflozin on liver fat in patients with type 2 diabetes and nonalcoholic fatty liver disease: a randomized controlled trial (E-LIFT Trial). Diabetes Care 2018;41:1801-8.

14. Eriksson JW, Lundkvist P, Jansson PA, et al. Effects of dapagliflozin and n-3 carboxylic acids on non-alcoholic fatty liver disease in people with type 2 diabetes: a doubleblind randomised placebo-controlled study. Diabetologia 2018;61:1923-34.

15. Sattar N, Fitchett D, Hantel S, et al. Empagliflozin is associated with improvements in liver enzymes potentially consistent with reductions in liver fat: results from randomised trials including the EMPA-REG OUTCOME® trial. Diabetologia 2018;61:2155-63.

16. Leiter LA, Forst T, Polidori D, et al. Effect of canagliflozin on liver function tests in patients with type 2 diabetes. Diabetes Metab 2016;42:25-32.

17. Kurinami N, Sugiyama S, Yoshida A, et al. Dapagliflozin significantly reduced liver fat accumulation associated with

Cite this article as: Scheen AJ. Effect of sodium-glucose cotransporter type 2 inhibitors on liver fat in patients with type 2 diabetes: hepatic beyond cardiovascular and renal protection? Ann Transl Med 2018;6(Suppl 1):S68. doi: 10.21037/ atm.2018.10.39 a decrease in abdominal subcutaneous fat in patients with inadequately controlled type 2 diabetes mellitus. Diabetes Res Clin Pract 2018;142:254-63.

18. Shimizu M, Suzuki K, Kato K, et al. Evaluation of the effects of dapagliflozin, an SGLT2 inhibitor, on hepatic steatosis and fibrosis by transient elastography in patients with type 2 diabetes and non-alcoholic fatty liver disease. Diabetes Obes Metab 2018. [Epub ahead of print].

19. Cefalu WT, Leiter LA, Yoon KH, et al. Efficacy and safety of canagliflozin versus glimepiride in patients with type 2 diabetes inadequately controlled with metformin (CANTATA-SU): 52 week results from a randomised, double-blind, phase 3 non-inferiority trial. Lancet 2013;382:941-50.

20. Ito D, Shimizu S, Inoue K, et al. Comparison of ipragliflozin and pioglitazone effects on nonalcoholic fatty liver disease in patients with type 2 diabetes: a randomized, 24-week, open-label, active-controlled trial. Diabetes Care 2017;40:1364-72.

21. Shibuya T, Fushimi N, Kawai M, et al. Luseogliflozin improves liver fat deposition compared to metformin in type 2 diabetes patients with non-alcoholic fatty liver disease: A prospective randomized controlled pilot study. Diabetes Obes Metab 2018;20:438-42.

22. Seko Y, Sumida Y, Tanaka S, et al. Effect of sodium glucose cotransporter 2 inhibitor on liver function tests in Japanese patients with non-alcoholic fatty liver disease and type 2 diabetes mellitus. Hepatol Res 2017;47:1072-8.

23. Akuta N, Watanabe C, Kawamura Y, et al. Effects of a sodium-glucose cotransporter 2 inhibitor in nonalcoholic fatty liver disease complicated by diabetes mellitus: Preliminary prospective study based on serial liver biopsies. Hepatol Commun 2017;1:46-52.

24. Li B, Wang Y, Ye Z, et al. Effects of canagliflozin on fatty liver indexes in patients with type 2 diabetes: a metaanalysis of randomized controlled trials. J Pharm Pharm Sci 2018;21:222-35.

25. Bajaj HS, Brown RE, Bhullar L, et al. SGLT2 inhibitors and incretin agents: Associations with alanine aminotransferase activity in type 2 diabetes. Diabetes Metab 2018. [Epub ahead of print]. 